\title{
Neurochemical markers in cerebrospinal fluid of patients with dementia George Paraskevas*
}

Address: Department of Neurology, Eginition Hospital, Athens University Medical School, Athens Greece

* Corresponding author

from International Society on Brain and Behaviour: 2nd International Congress on Brain and Behaviour Thessaloniki, Greece. 17-20 November 2005

Published: 28 February 2006

Annals of General Psychiatry 2006, 5(Suppl I):S58 doi:I0.II86/I744-859X-5-SI-S58

The presence of a long history of alcohol abuse in patients with cognitive decline may be a problem in the differential diagnosis, especially when dealing with elderly patients, where Alzheimer's disease (AD) is the most frequent cause of dementia. Since specific treatments are now available for $\mathrm{AD}$, while the effect of alcohol on cognition may be reversible to some extent, the need for early discrimination between the two disorders is obvious.

Cerebrospinal fluid (CSF) markers including tau protein, amyloid $\mathrm{A} \beta 42$ and their ratio received much attention recently, since they may be of some help in the diagnosis of $\mathrm{AD}$ and the differential diagnosis from other causes of dementia, in terms of every-day practice. In the differential diagnosis of $\mathrm{AD}$ from alcohol-related cognitive decline CSF tau is better than $A \beta 42$ alone. In a patient with cognitive decline and history of alcohol abuse increased levels of CSF tau almost confirm the (co)existence of $\mathrm{AD}$ at all levels of clinical confidence. However, normal CSF tau levels do not always exclude AD. The combined use of tau with $A \beta 42$ in the form of their ratio (tau/A 442$)$ resolves the problem. An increased ratio almost confirms the (co)existence of $\mathrm{AD}$, with a positive predictive value that may approach or even exceed $99 \%$ at all levels of clinical (pre-test) confidence, while a normal ratio almost excludes the (co)existence of $\mathrm{AD}$, with a negative predictive value $>90 \%$ at all levels of clinical confidence. The discriminating value is comparable in patient with early cognitive decline (MMSE > 23) or more advanced disease.

The method required for the determination of tau and Aß42 is an ELISA method, which is easy to perform and does not require ionizing radiation, specially trained personnel and expensive equipment. Lumbar puncture is a minimally to moderately invasive procedure, but usually well tolerated and safe, provided that there is no contraindication. The incidence of post-lumbar puncture headache is low in the elderly population and probably no more than $5.5 \%$. Given the above, the determination of CSF markers of $\mathrm{AD}$ may be helpful in the every-day diagnostic work-up of elderly demented patients with a history of alcohol abuse. 